\title{
ON THE STUDY OF DISEASES OF THE NERVOUS SYSTEM.
}

BY J. HUGHLINGS JACKSON, M.D.

A Lecture delivered June, 1864.

(Reprinted from the London Hospital Reports, vol. i., 1864, at the special request of Sir William Broadbent.)

Gentlemen,-_" It is easier," Lord Bacon says, " to evolve truth from error than from confusion." If we look back, most of us would say of our mental culture that the great want was not a want of industry, nor of opportunities, but of method. And in the study of disease it is better to have even a mechanical arrangement than to work without a plan. But I hope that the method I am about to submit to you is in some sense a natural system, rather than an artificial scheme. If you find it helps you keep it; if it trouble you reject it. Each has his peculiar mental temperament, and a plan which to one is a help would be a constraint to another. I think the value of my method is that it will prevent your mistaking a complete view of one aspect of a case for a knowledge of the patient's disease. Thus, you may study the physiology of a case minutely and accurately, but this is not the disease. You may study the clinical history and neglect the physiological; this also is an imperfect foundation for thinking of a patient's case. You will see better what I mean after a few illustrations. I will now simply repeat what I have said, that neither the physiological history alone, nor the clinical history alone, is to be taken as a basis for our diagnosis, or as a basis of treatment. It is from a consideration of both, that 
we arrive at the natural history and act for our patient's advantage in diagnosis, prognosis, and treatment.

But I must insist on one thing as an essential preliminary in the study of disease, and markedly essential in the study of diseases of the nervous system, viz.: a knowledge of anatomy. Try to explain to anyone who does not know anatomy, from disease of how many different cranial nerves sight may be affected. It would be as hopeful to try to explain to a blind man what the colour blue was like. For we must, in studying diseases of the nervous system, be precise and not merely minute. I believe, and I hope I shall give you some reasons for my belief, that diseases of the nervous system are not more obscure than those of the chest or abdomen. It is the anatomy that is difficult. It is a truism to say that we cannot tell the value of "strabismus" as a symptom in brain disease if we do not know what nerves supply the recti ; and for want of knowing this, we may be wrong in our diagnosis of the seat of disease in the central nervous system.

Our pathology should be both particular and general. We should try to make it as wide in its principles as our physiology will let us, but at the same time as precise and accurate as our anatomical knowledge will permit. Just as we study, as physiologists and anatomists, the vegetative life of general tissues, the structure of organs for special functions, and the universal harmony of most diverse functions in individuals, so we ought, as workers in the field of practical medicine, to study every case that comes before us; as presenting :-

(1) Disease of Tissue. (Changes in tissue.)

(2) Damage of Organs.

(3) Disorder of Function.

Learn the tissue health of a patient, as shown by the colour of his hair, the elasticity of his skin, the tint of his complexion, the feel of his arteries, and the condition of his urine. Examine the chief organs, and with especial care the one in fault, or supposed to be in fault; and 
lastly, be most careful to ascertain if organs near or distant to the one or more most damaged act in harmony -if function be disordered. Of course, disease of tissue damages the organ of which it forms part, and disorders its function, but that special tissue may be diseased or liable to it in nearly every other part of the body, and again the damaged organ may disorder the functions of most distant parts.

My lecture will be chiefly a series of recapitulations of the above general views by special instances. I prefer to take as my first illustration a common general disease which produces much local damage to several organs, viz., rheumatic fever. This disease is exceedingly common at this Hospital, and as a consequence, valvular disease of the heart and chorea are also common, and now and then as a further consequence, embolism. I take this illustration in preference to one of disease of the nervous system, as a method, to have any real root in nature, should apply to diseases and diseased conditions generally and not to a limited nosological group.

Rheumatic fever is a blood disease, in which the fibrous tissues are attacked. The pericardium and the endocardium suffer, and the valves of the heart especially suffer as they contain a good deal of this tissue. Next, as a consequence of the disease of fibrous tissue in these valves, the heart (the organ) is damaged as the segments of its valves do not fit, and thirdly, as the heart is the central organ of the circulatory system, the functions of all other parts, but especially those of the lungs and liver, are liable to be disordered. Now, notwithstanding the importance of the organ affected, we have in rheumatic fever to think more of inflamed fibrous tissue than of heart disease; and the question as we stand by the bedside ready to prescribe, is how to prescribe for a patient who has rheumatic inflammation of his fibrous tissues, and just then the information as to the precise valve affected is comparatively insignificant.

VOL. XXVI. 
I am aware that some would treat inflamed fibrous tissue in the valves of the heart with greater energy than they would similar disease in less important parts, as it is desirable at even great risk to get rid of lymph effused in fibrous tissue so importantly situated. It is just as a general in war will defend a point, not on account of its size or intrinsic importance, but on account of its strategetical value. The strategetical value of the flaps of fibrous tissue (the mitral valves), between the left auricle and left ventricle is very great.

Suppose the turmoil of tissue changes is over, and that the patient has got "well," as he supposes, and perhaps even as we thought too. A year later, he comes to us again, and we find that he is suffering from disease of the heart. We must now think most seriously as to the kind of damage to the organ. For, although the damage is exceedingly local, it is in an essential part of the mechanism of a most central organ. We get to know generally the kind of damage by auscultation and by percussion. Still we cannot treat a damaged organ as a surgeon can; he can make an artificial pupil, or nail two bones together, but when tissue changes are over, we can do little to reverse them. We must pass next to the disorder of function. The patient often does not know that he has disease of the heart, but comes because he has dropsy or breathlessness. He does not come for his damaged organ, but for disorder of function consequent on derangement of its mechanism. We may find a bruit when a patient attends for some other disease, but we can do nothing for that. We can never make the valves fit, nor remove vegetations from them; so that it is of more moment to study the functions disordered, which we can help, than the organ damaged which we cannot repair. But information about the latter is of great value, as it helps us to gain a clear idea of the former.

Here let me give you a piece of advice. Never treat a patient on stethoscopic evidence only. The heart may be a bad musical instrument and yet a good force-pump. 
However noisy the first sound may be at the base of the heart, if the apex be in its right place, if there be no signs of hypertrophy, no anæmia, if the pulse be good, and, above all, if the patient does his work well, we have nothing to treat. To treat such a case would be to treat a sound. It would be just as great a mistake as the drunken captain made, who tried to navigate his vessel round a speck of dirt on his chart, which he mistook for an island. ${ }^{1}$ And in cases of aortic or mitral regurgitation, we could only at the most give such remedies as would improve the general health, or possibly act on the muscular tissue of the heart itself to enable it to keep up the stream of blood in spite of the imperfection of its valves. But we should render the patient much more service by advising him how a man with a damaged heart should choose his occupation, so that the circulation of important organs might not be obstructed, or in other words, that function might not be disordered.

The best illustration I can give you of disorder of function in heart disease is the pneumonia, so-called, of mitral obstruction. It is frequently the immediate cause of death in heart disease. But see how different this disorder of function is from real pneumonia, in which the tissues of the lung are affected. In one the lung's business is obstructed, in the other its texture is diseased. The congestion of the first is because the blood cannot get out of the lungs, from obstruction to the column at the mitral orifice; of the other, because it is held there by the tissues. And after the stage of congestion, there is still a perceptible difference in the progress, the first tending to so-called apoplexy of the lung and hæmoptysis, and the other to effusion of lymph into the air-cells, and to hæmoptysis too, but of a different kind.

" Generally spesking, I would say that the tendency of half-instructed ausculators is to over-estimate the importance of the murmur as a fact, and to under-estimate it as a means of investigation; to pay too much attention to the mere existence of the sound, and too little to the circumstances in which it occurs."-Gairdner, Clinical Medicine, p. 561. 
Fatty degeneration of the heart, the hypertrophy of the left ventricle, found so often in chronic Bright's disease, and disease of the aortic valves, say from hard work, as hammering, are all " diseases of the heart." But, as I hope to show you when I speak of diseases of this organ, in their clinical association with diseases of the nervous system, the significance of each for diagnosis and treatment is very different to that of the others. In all the same organ is damaged, but they are diseases of different tissues, and, as I think, produce different symptoms, i.e., disorder differently the functions of other parts or organs.

In the eye, in the organisation of which most of the tissues of the body are used, we may find plenty of illustrations of the method I have mentioned. And here I may say that the study of disease of tissues may be carried on with greater advantage in the eye than in any other part of the body. We can watch the changes in a nodule of lymph on the iris, and can see the retinal degeneration attending chronic Bright's disease. Here only lies a nerve termination, with an artery and a vein, under our view.

We should all study diseases of the eye if we wish to know diseases of the nervous system, and indeed pathology generally. Besides the importance of this knowledge as a help to the study of the physiology of disease (for six of the nine cranial nerves have to do directly or indirectly with the organ of vision), it is a field for the study of diseases of tissues. It is, I think, of quite as much importance as a practical knowledge of the chemistry of the urine. In passing we may remark that it is quite erroneous to suppose that the introduction of the stethoscope, ophthalmoscope, and laryngoscope has made our medical lives more laborious. On the contrary, we have more things to do, but we do our work more easily, and far more satisfactorily.

Now, in the eye, it is of infinitely more importance 
to recognise the early stage of disease of tissue-the stage of change-than to learn the exact part of the organ of vision damaged. But I admit that we often can in eye diseases, by the ophthalmoscope, detect both the tissue changes and the part of the organ where they are, and often by empirical experience tell the constitutional cause of the disease: It is, however, of importance to study diseases of the eye for other purposes, even when the active changes are over, for their value as showing the particular tissues undergoing change. Thus, syphilitic choroiditis and syphilitic inflammation of the pia mater -the choroid of the brain-are pretty much alike as diseases of tissue, although they produce very unlike physiological symptoms, as the organs they damage, and the functions disordered are very different. If I may use such expressions in pathology, the "idea" is the same, but the "events" are different. See the practical bearing of this. A patient comes to us with acute pain limited to a spot on one side of his head, and partial "amaurosis." Now, if we find the "amaurosis" to depend on syphilitic choroiditis, the information is most valuable, however old the choroid disease may be, and however hopeless the treatment of that might now be. For it would give us good grounds for hope that the acute limited pain on one side of the head was the sign of a new syphilitic inflammation of a similar tissue, the pia mater, and that our treatment in the stage of change might save the brain from damage, although it might do no good to the older disease of the choroid which (like mitral valves, damaged by a bygone attack of rheumatism) had passed through the stage of change, in which we may help, to one of permanence, the effused products having, in fact, become a bastard tissue, and only in degree less vital than the legitimate tissues.

I will take a few more illustrations from the eye. Choroiditis and retinal apoplexy are alike as damages of organs, but different as diseases of tissue. Presbyopia 
and paralysis of the ciliary muscle are pretty much alike as disorders of function, but very different as diseases of tissue. Thus, the latter is due to disease of the third nerve or to that part of it which supplies the ciliary muscle, whilst the former is chiefly a change (in the firmness of the lens) of advancing years. And it is well to remember this as a fact, and I mention it to you as I was once puzzled because I did not think of it. A patient seventy years of age had paralysis of the third nerve on one side, but the sight on the paralysed side was quite as good as on the other. The reason of this apparent exception was that the accommodation of both eyes had failed long before, and paralysis of the ciliary muscle could not make them worse. He was already completely presbyopic, so that really instead of saying the eyes were equally good, we should say in such a case that they were equally bad.

In an organ like the ear about which medical men, or physicians at least, generally know little, it is obviously of more importance to ascertain that the patient is suffering arthritically or syphilitically, than to get to know what is the exact part of the organ which is damaged.

In diseases of nerve trunks, see how exceedingly different are the physiological symptoms (the disorders of function) of disease of the optic, the portio dura and the ulnar nerves, although they may be quite, or closely, alike as diseases of tissue. A nerve trunk besides its nerve tubes consists of fibrous and connective tissue. It is compound, and in a loose sense is an organ. Suppose it damaged by syphilis, as in syphilitic neuroma. Here you see the important fact to be known is, that the patient has syphilitic disease of fibrous and connective tissue, an exact knowledge of the individual nerve-bundle attacked and the functions disordered being of secondary importance.

The transition from nerve-trunks to the central nervous system is easy. Never forget that every organ is made up of constituents which exist more or less in the greater part of the body. We have in nervous masses, 
nerve-cells and fibres, blood-vessels, and also connective tissue. So, too, we find that "Diseases of the Nervous System" are not always diseases of nervous tissue; but often of other tissues which enter into the composition of nervous organs-of blood-vessels, connective tissue, \&c. Now, it is a well-known fact, that syphilitic deposits are generally found on the surface of the brain. They begin in the pia mater and extend into the nervous mass; in short, they affect the connective tissue where it is in bulk. There is, although this is denied too, connective tissue in the substance of the brain itself, but I never saw deposits unconnected with the pia mater; the reason being probably the simple one that there is very little, if any, connective tissue in the central masses of the nervous system.

It may seem contradictory to say that I have no experience of syphilitic diseases of nervous matter, when in the next breath I speak of syphilitic epilepsy and syphilitic paralysis. But you will readily see that it is really important to know whether the epilepsy be due (directly or indirectly) to disease of the connective tissue in or on the nervous masses or to some change in the nerve-fibres or nerve-cells themiselves. We cannot set to work to treat an organ like the corpus striatum, but we may prescribe for a patient who has syphilitic disease of connective tissue, wherever it may be. Now syphilitic deposit in the pia mater is essentially the same as a node of the tibia, or a cellular node of the skin, or of the spleen and liver, and it requires the same treatment as these. I cannot too much impress on you that we ought above all things, in brain disease to study diseases of tissues. It is of infinitely more importance than to know the exact organ of the brain damaged; for instance, to know that there is lymph effused in the pia mater, than that the attendant epilepsy, the disorder of function of part of the brain, has certain physiological peculiarities. Indeed, for my part, I fear we are too late, for cure at 
least, when the epilepsy is well established. Then, the tissue changes in the pia mater have settled down, the brain is damaged, and its functions will continue to be disordered more or less in spite of what we can do. It is in the stage of inflammation, when, as I have before said, the patient comes with pain on one side of his head that we may help, not when he is "well" of this and a few months afterwards comes for epileptiform seizures affecting the opposite side of the body. It is when the pia mater is in a condition analogous to acute iritis and not in one analogous to occluded pupil from bygone inflammation of the iris.

Now for still more illustrations of these principles, and I hope I am also teaching you some of the details of brain disease in giving them. You may, perhaps, say that you cannot help, instinctively, carrying on the three trains of thought in investigating a case. Now I deny this. A little observation will tell you it is incorrect. Do we not sometimes treat convulsions by specifics, with too little thought as to the exact part of the brain damaged and as to the real tissue diseased, and, more broadly, do we always take the condition of the patient into equal consideration in thinking of his disease? Indeed, the paroxysm, the disorder of functions, is often taken for the whole disease when it is only the outward part of it by which we ought to try to learn the rest. It is not at all a rare thing to hear of a patient, after his first attack of epilepsy, being treated as if the convulsions were a sign of inflammation-disordered function being taken as evidence of tissue changes, of inflammation or congestion. Now, after a severe fit of epilepsy, and especially after a series of fits, there is a kind of congestion, but it is no more related to inflammation than the pneumonia of heart disease is to true pneumonia. There is too much blood in the vessels of the brain; but it is there because it cannot get along, and not because it is held by the tissues. Here then let me impress on you not to take the 
most violent symptoms of disordered function of the brain for inflammation, and remember this especially in injuries to the head. In children it is notorious that convulsions frequently signify just the reverse-debility. A rigor, which may attend suppuration anywhere, is far more valuable information that tissue changes are taking place in the head than convuls.ons. Now remark, I here give no opinion as to whether you should bleed or not in either the condition of congestion of disordered function, or in the genuine congestion of inflammation. All I say is this, know what you are about. What you do, do advisedly, and do not bleed by routine for "head symptoms," because the organ damaged is an important one.

To think of a case as one of convulsions, or as epilepsy, without reference to the tissue changes and the organ really in fault, is a very careless way of thinking. The epilepsy of a young man coming on gradually, and persisting for years, is generally quite a different disease to that of a man of middle life with rigid arteries and signs of degeneration, although the convulsion, the disordered function, may be quite alike. In a case like this, how very foolish we should look if we had diagnosed brain disease. A young man supposed to be quite healthy, falls down in convulsions. He soon becomes sensible again, but his urine is found to be bloody and albuminous, and it is ascertained that several of his brothers and sisters are then suffering from scarlet fever. Here, obviously, the disease was disorder of function of the brain, and the organ most anatomically changed was one remote - the kidney.

I have seen a patient treated for delirium as if it were due to "congestion" of the brain, with no benefit, who was sent to sleep at once by brandy and water. It was common at one time to treat mania as if it were due to inflammation of the brain; but in the last case I saw, although the patient was furious, bottled stout and brandy were given, and with benefit. 
How very different is true croup which occurs in a healthy, perhaps in a robust, child, and which impedes the entrance of air by disease in the larynx, from false croup, which comes on in a ricketty child, and impedes respiration by partially closing the glottis. (By the way, false croup is generally called "fits" by the nurse.) One is disease of the tissues of the organ, and the other disorder of a mechanical function. Here the treatment is obviously quite different. Then, too, how often has disorder of function of the larynx, from pressure on the recurrent laryngeal by a tumour in the thorax, been taken for disease of the larynx itself.

Let me repeat then for the last time, before my final illustrations, that in each case we must study changes in tissues, examine the organs damaged, and investigate the disorders of function. But remark, although inseparable, each of the three trains of thought ought to be distinct, or the more information we get the more confused we shall be. Let me illustrate this. In a cerebral case you examine the eyes, and record the following facts : The patient has an arcus senilis, a large pupil, paralysis of the sixth nerve, white atrophy of the optic nerve, and the retinal degeneration of Bright's disease. Now, it might be a nice arrangement to put all these things down under the heading of "eye symptoms," and, indeed, as an index, might be useful. But the student who thought of these different symptoms from one point in his own mind would have learnt many things, but would after all know little. He might be very minute, but would never be precise. He would attend to the superficial contiguity of events, and neglect the more real continuity of ideas. Such a man's classifications and arrangements would have the merit of neatness, but he himself would have a most unmethodical mind. There would be a superficial appearance of order, but a real incoherence. I think this kind of order is the very worst form of slovenliness. For some of these eye symptoms are merely local 
signs of almost universal changes. Let us never forget than an elaborate arrangement is often the highly-worked cloak that covers up inferiority of organisation. The most gorgeous piece of mosaic is not equal to the simplest natural flower. The mere minuteness that lacks precision is always ready to deal in generalities, but never arrives at a scientific generalisation. Of what value would be our minute inquiries as to convergent strabismus if we did not distinguish whether it was due to paralysis of the external rectus or to hypermetropia? Indeed, the more minute a man is who does not think of his anatomy, or who studies merely one side of disease, the more harm will he be likely to do to the science of practical medicine.

I take hemiplegia as the last illustration, and, for ease of description, hemiplegia on the left side. Hemiplegia is the simplest, the best known and the commonest disease of the nervous system.

(1) A patient may have hemiplegia from syphilitic disease of the pia mater on the surface of the right hemisphere. $^{1}$

(2) A patient may have hemiplegia from embolism of the right middle cerebral artery.

(3) Because blood has been effused in the right corpus striatum.

(4) Because a tumour has grown in, or grown into, the right corpus striatum.

Now, in all these cases, the hemiplegia would be of the same kind. We might choose four cases in which the physiological symptoms would be alike, even in degree, to a nicety. For the organ damaged, the corpus striatum, would be, so far as the hemiplegia is concerned, just the same.

'Although the obvious disease is found here, there can be little doubt that the hemiplegia is due to some secondary change in the motor tract. There is no fact better established clinically, than that very extensive disease of the hemisphere may exist without any hemiplegia. I shall, in a future communication, give my reasons for believing that disease on the surface of one hemisphere, when it does cause hemiplegia, does so by producing disordered circulation in an arterial region-that of the middle cerebral artery. This vessel supplies the corpus striatum, as well as the part (the hemisphere) on the surface of which is the most obvious disease. 
But to think of these cases baldly under the name of hemiplegia, as an entity, would leave us with very vague grounds for treatment. We should be on a par with the public, who ask what is good for a cough? In the first case, we should have to treat syphilis; in the second, limited softening of the brain; in the third, some more general affection, of which degeneration of arteries was the most prominent feature, and rupture of one or more vessels in the corpus striatum the most dramatic event; and in the last, tumour, if we knew how. In all, we should treat the patient.

If, then, a patient comes to us with hemiplegia, we study him as well as his local disease-his tissue changes and general health, as well as his paralysed arm and leg. If he be past fifty, if he have rigid arteries, an arcus senilis, if he have Bright's disease, and above all, if the attack were sudden (although it might not be suddenly complete), we diagnose effusion of blood in nervous tissue. But if he be a young man having healthy tissues, and if the attack began in his usual health, we ought to see if he have not valvular disease of the heart, and ask if he has had rheumatism or scarlet fever. If it began slowly, or if it followed an attack of unilateral convulsions, and especially if preceded by pain in the opposite side of the head, we should seek for evidence of syphilis. (Here I may just say that the clinical history of the epilepsy and paralysis from syphilis is like that of the same diseases which follow injury to one side of the head, in this, that the convulsions and the paralysis are generally quite unilateral.) To recapitulate: (1) syphilitic deposit on the surface of the hemisphere; (2) softening from plugging of an artery; (3) blood effused in nervous tissue; and (4) a new growth.

Having given you instances of damages of the same organ by different affections of its tissues, I will now give you instances of damages of very different organs, the result of similar tissue changes. The following four cases 
of hemiplegia are each of a different and well-marked kind from disease of the same sort, but at different points in the motor tract:-

(1) A patient has suddenly paralysis of the left arm and leg, and of a very small part of the face, and slight deviation of the tongue. Clot in the right corpus striatum.

(2) Paralysis of the third nerve on the left side, and of the arm and leg and of a small part of the face on the right. Clot in the left crus cerebri.

(3) Paralysis of the portio dura, and complete deafness on the left side, paralysis of the arm and leg on the right. Clot in the floor of the fourth ventricle involving the nuclei of the left facial and auditory nerves and also the fibres which have just crossed coming from the limbs on the right side.

(4) Sudden and complete paralysis of the tongue, palate, and of the vocal cords all on the left side, weakness of the left limbs, and slightly of the right. Clot, involving the lingual and spinal accessory nuclei, and implicating the fibres from the limbs, about their decussation.

Now, how exceedingly various are the sets of symptoms produced by the same tissue changes. But we have to think, as regards treatment, of effusion of blood in nervous tissue, rather than of disease of the corpus striatum, crus cerebri, pons varolii, \&c. To attend to the seat of the damage only is to study merely the physiology of disease, and is not practical medicine.

I should be misunderstood if I were supposed to underrate the physiological study of diseases of the nervous system. Indeed, I think that to neglect it shows want of education, but to neglect the clinical shows want of experience and sagacity. Never forget that we may run the risk of being over-educated and yet under-cultivated. We may be gorged with facts in anatomy, physiology, and pathology, and yet our faculties may be little trained for investigating disease. Sometimes the physiological history and the clinical history point in the same direc- 
tion. Thus, paralysis of part of the face, with slight defect of articulation, shows central disease; and this information, with evidence of wide tissue degeneration, points to the conclusion that our patient has not only a degenerated nervous system, but that it is now breaking up. Again, a patient (the last of the four cases just given) has paralysis of the palate and of the tongue, and of the internal muscles of the larynx on one side. This is physiological evidence that he has disease, as Lockhart Clarke's researches clearly show, at the nuclei of the lingual and spinal accessory nerves. This, then, shows that the disease is in a dangerous position, for although it may be local it is very central, and, besides, perilously near the socalled vital knot. So much for the physiological history of the case; and now for the clinical. The patient has albuminous urine, but no dropsy. He has, too, the degeneration of the retina found so often in chronic Bright's disease, or, as it might be vaguely called, "partial amaurosis." So, then, he has already central disease, in the medulla oblongata, and he has, too, signs of wide degeneration of general tissues, which render him liable to further damage in other parts of the nervous system.

In future lectures we shall study individual cases more closely. 